\title{
A Bag Theoretic Approach towards the Count of an Intuitionistic Fuzzy Set
}

\author{
${ }^{1}$ B.K.Tripathy, ${ }^{2}$ S.Khandelwal \\ School of Computing Science and Engineering, VIT University, Vellore -632014, Tamil Nadu, India \\ Email: ${ }^{1}$ tripathybk@vit.ac.in, ${ }^{2}$ subhamkhandelwal@yahoo.co.in \\ M.K.Satapathy \\ Department of Mathematics, R.G. College, Padmapur, Odisha, India \\ Email: msat141106@gmail.com
}

\begin{abstract}
The cardinality of fuzzy sets was introduced by DeLuca and termini, Zadeh and Tripathy et al, where the first one is a basic one, the second one is based on fuzzy numbers and the final one introduces a bag theoretic approach. The only approach to find the cardinality of an intuitionistic fuzzy set is due to Tripathy et al. In this paper, we introduce a bag theoretic approach to find the cardinality of intuitionistic fuzzy set, which extends the corresponding definition of fuzzy sets introduced by Tripathy et al. In fact three types of intuitionistic fuzzy counts are introduced and we also establish several properties of these count functions.
\end{abstract}

Index Terms - Fuzzy Set, Intuitionistic Fuzzy Set, IFGCount, IFLCount, IFECount, Bags.

\section{INTRODUCTION}

The notion of sets introduced by Cantor is fundamental to crisp mathematics. However, it was found that its modeling power is limited as real life situations are not crisp. Thus models which can handle uncertainty data needed to be developed. Thus, the introduction of the fuzzy set concept by Zadeh [1] is considered as a paradigm shift [2]. It introduces the concept of graded membership of elements instead of the binary membership of elements in a crisp set. It is a very powerful modeling language that can cope with a large fraction of uncertainties of real life situations. Because of its generality it can be well adapted to different circumstances and contexts.

The cardinality of a set in the crisp sense plays an important role in mathematics and its applications. Similarly, it is worthwhile to think of cardinality of fuzzy sets, which is a measure. The concept of cardinality of a fuzzy set is an extension of the count of elements of a crisp set. A simple way of extending the concept of cardinality was suggested by Deluca and Termini [3]. Another approach to define the cardinality of a fuzzy set which depends upon the probability measure of a fuzzy set is due to Zadeh [4] and is termed as the sigma count or the non-fuzzy cardinality of a set. One of the most natural ways of defining the cardinality of a fuzzy set is due to Zadeh [5] in which the concept of fuzzy number introduced by Mizumoto et al [6] has been used.
According to fuzzy set theory, the non-membership value of an element is one's complement of its membership value. However, in practical cases it is observed that this happens to be a serious constraint. So, Atanassov [7] introduced the notion of intuitionistic fuzzy sets as a generalization of the concept of fuzzy sets. The only definition of the count of an intuitionistic fuzzy set proposed so far is due to Tripathy et al [8], which is in the direction of Deluca and termini [3]. The notion of crisp set has two limitations. The first one is that the repeat ion of elements is not permitted and the second is that the order of elements is not important. In an attempt to remove the first constraint, that is to permit multiple occurrences of elements in a collection, the concept of bag or Multiset [9, 10, 11, 12] was introduced and as expected it has better modeling power than a set. For example, telling the number of copies of books in a store provides better idea than simply telling which books are there in it. In this paper, we introduce another approach to the count of an intuitionistic fuzzy set, which follows the bag theoretic approach of Tripathy et al [13] in defining three types of fuzzy counts. We establish many properties of these three fuzzy counts and find that some of the properties in the base case do not hold true in the generalized case.

The structure of this paper is as follows. In the next section we present the notations and definitions to be used in the paper. In section 3, we introduce our notion of intuitionistic fuzzy counts using the bag theoretic concept. In section 4, we establish some properties of these intuitionistic fuzzy counts. We follow it with a conclusion in section 5 and the list of references in section 6 .

\section{DEFINITIONS AND NOTATIONS}

In this section, we shall provide some definitions and notations to be used in this paper.

Definition 2.1: Let $X$ be a universal set. Then a fuzzy set $\mathrm{A}$ on $\mathrm{X}$ is defined through a membership associated with $\mathrm{A}$ and is denoted as

(2.1) $\mu_{A}: X \rightarrow[0,1]$, 
such that every $x \in X$ is associated with its membership value $\mu_{A}(x)$ lying in the interval $[0,1]$.

Clearly a fuzzy set A is completely characterized by the set of points $\left\{\left(x, \mu_{A}(x)\right): x \in X\right\}$.

In a fuzzy set as defined above it is assumed that the non-membership function is always equal to the one's complement of the membership function. That is the nonmembership values of the elements can be obtained by subtracting the membership value from one.

But in many real life situations it can be seen that this is not the case. For example, in the exit polls, besides the "yes" and "no" options there is a third option called "can't say". The elements in the last category are uncertain about their decision. Hence, in this case the membership of an element into the "yes" category cannot be equal to one's complement of its non- membership, which is membership in the "no" category.

Bearing these types of examples the notion of intuitionistic fuzzy sets was introduced by Atanassov in 1986 [7], which is formally defined as follows.

Definition 2.2: Let $X$ be a universal set. An intuitionistic fuzzy set (IFS) $\mathrm{A}$ on $\mathrm{X}$ is defined through two functions $\mu_{A}, v_{A}$, called the membership and nonmembership functions of A respectively and defined as

(2.2) $\mu_{A}: X \rightarrow[0,1]$ and $v_{A}: X \rightarrow[0,1]$,

such that every $x \in \mathrm{X}$ is associated with its membership value $\mu_{A}(x)$ and the non-membership value $v_{A}(x), \quad$ satisfying the condition, $0 \leq \mu_{A}(x)+v_{A}(x) \leq 1$.

An intuitionistic fuzzy set $\mathrm{A}$ is represented by the set of points $\left\{\left(x, \mu_{A}(x), v_{A}(x)\right): x \in X\right\}$.

There is an important concept associated with every intuitionistic fuzzy set called the hesitation function. For every $x \in X$, its hesitation value is denoted by $\pi_{A}(x)$ and is given by

$$
\pi_{A}(x)=1-\mu_{A}(x)-v_{A}(x) .
$$

For a fuzzy set the hesitation for any element in the set is zero. So, obviously when $\pi_{A}(x)=0$ or equivalently $v_{A}(x)=1-\mu_{A}(x)$ an intuitionistic fuzzy set reduces to a fuzzy set.

Definition 2.3: If $A$ and $B$ are two IFSs of the set $X$, then

(2.3) $A \subseteq B$ iff

$\forall x \in X, \mu_{A}(x) \leq \mu_{B}(x)$ and $v_{A}(x) \geq v_{B}(x)$

(2.4) $A \subset B$ iff $B \subset A$

(2.5) $A=B$ iff $\forall x \in X, \mu_{A}(x)=\mu_{B}(x)$ and $v_{A}(x)=v_{B}(x)$

(2.6) $A^{C}=\left\{\left(x, v_{A}(x), \mu_{A}(x)\right): x \in X\right\}$

(2.7) $A \bigcap B=$

$\left\{\left(x, \min \left(\mu_{A}(x), \mu_{B}(x)\right), \max \left(v_{A}(x), v_{B}(x)\right)\right): x \in X\right\}$

(2.8) $A \cup B=$

$\left\{\left(x, \max \left(\mu_{A}(x), \mu_{B}(x)\right), \min \left(v_{A}(x), v_{B}(x)\right)\right): x \in X\right\}$

(2.9) $A+B=$

$\left\{\left(x, \mu_{A}(x)+\mu_{B}(x)-\mu_{A}(x) \cdot \mu_{B}(x), v_{A}(x) \cdot v_{B}(x)\right): x \in X\right\}$

(2.10) $A \cdot B=$

$\left\{\left(x, \mu_{A}(x) \cdot \mu_{B}(x), v_{A}(x)+v_{B}(x)-v_{A}(x) \cdot v_{B}(x)\right): x \in X\right\}$

As mentioned above, number of times an element occurs in a set has no meaning or in other words we do not allow repetition of elements here. However, in real life situations, repetition of elements is significant as explained through an example in the introduction. A collection of distinguishable elements where repetition of elements is allowed is called as a "bag" or a "multiset".

This notion was introduced by Cerf et al ([9]) in 1971, Peterson in 1971 ([11]) and Yager in 1986 [12]. These authors have mostly used the term "bag" to address this model. Several properties of bags have been established by these authors. This study was further carried out by Chakraborty et al ([14]). We formally define it as follows.

Definition 2.4: A bag $B$ drawn from a set $X$ is represented by a function "Count $\mathrm{B}$ " or $C_{B}$ and is defined as

$$
C_{B}: X \rightarrow N
$$

where $\mathrm{N}$ denotes the set of non-negative integers.

To be precise, for any element $x \in X$ the $C_{B}$ function associates with the value $C_{B}(x)$, which is the number of times $\mathrm{x}$ occurs in B.

Some basic properties of bag are as follows:

(i) A bag B is a set if $C_{B}(x)=0$ or 1 for all $x \in X$

(ii) The support set $B^{*}$ of $\mathrm{B}$ is defined as

$$
B^{*}=\left\{x \in X \mid C_{B}(x)>0\right\}
$$

(iii)For two bags $B_{1}$ and $B_{2}$ drawn from a set $X$, we say that $\mathrm{B}_{1}$ is a subbag of $B_{2}$ and write $B_{1} \subseteq B_{2}$ iff $\forall x \in X, C_{B_{1}}(x) \leq C_{B_{2}}(x)$.

(iv)Two bags $B_{1}$ and $B_{2}$ are said to be equal iff $B_{1} \subseteq B_{2}$ and $B_{2} \subseteq B_{1}$ and we write $B_{1}=B_{2}$.

(v) The cardinality of a bag $\mathrm{B}$ drawn from $\mathrm{X}$ is denoted by $\operatorname{Card}(\mathrm{B})$ and is given by

$$
\operatorname{Card}(B)=\sum_{x \in X} C_{B}(x)
$$


whenever the right hand side is finite or convergent. For the sake of convenience we denote $\mathrm{Card}(\mathrm{B})$ by $|\mathrm{B}|$.

(vi) The insertion of an element ' $\mathrm{x}$ ' into a bag $\mathrm{B}$ results in a new bag B' which is denoted by $B \oplus x$ such that

$$
\begin{aligned}
& C_{B^{\prime}}(x)=C_{B}(x)+1 ; \\
& C_{B^{\prime}}(\mathrm{y})=C_{B}(\mathrm{y}), \text { otherwise. }
\end{aligned}
$$

(vii) The removal of an element ' $\mathrm{x}$ ' from a bag $B$ results in a bag $\mathrm{B}^{\prime}$, denoted by $B^{\prime}=B \Theta x$ such that

$$
\begin{aligned}
& C_{B^{\prime}}(x)=\max \left\{C_{B}(x)-1,0\right\} ; \\
& C_{B^{\prime}}(\mathrm{y})=C_{B}(\mathrm{y}), \forall \mathrm{y} \neq \mathrm{x} .
\end{aligned}
$$

(viii)If $\mathrm{B}_{1}$ and $\mathrm{B}_{2}$ are two bags drawn from a set $\mathrm{X}$ then the removal of the bag $B_{2}$ from the bag $\mathrm{B}_{1}$ results in a bag $\mathrm{B}$, denoted by $B=B_{1}-B_{2}$ such that

$$
\begin{aligned}
& \forall x \in X, \\
& C_{B}(x)=\max \left\{C_{B_{1}}(x)-C_{B_{2}}(x), 0\right\}
\end{aligned}
$$

(ix) Two bags $B_{1}$ and $B_{2}$ are said to be equivalent iff $|\mathrm{A}|=|\mathrm{B}|$.

Definition 2.5: Let $B_{1}$ and $B_{2}$ be two bags defined over a universe $\mathrm{X}$. Then for any $x \in X$, we have the following

$$
\begin{aligned}
& \text { (2.11)If } \mathrm{B}=B_{1} \bigcap B_{2} \\
& \text { then } C_{B}(x)=\min \left\{C_{B_{1}}(x), C_{B_{2}}(x)\right\} \text {. } \\
& \text { (2.12)If } \mathrm{B}=B_{1} \cup B_{2} \\
& \text { then } C_{B}(x)=\max \left\{C_{B_{1}}(x), C_{B_{2}}(x)\right\} \text {. } \\
& \text { (2.13)If } \mathrm{B}=B_{1} \oplus B_{2} \text { then } C_{B}(x)=C_{B_{1}}(x)+C_{B_{2}}(x)
\end{aligned}
$$

The following definition of the cardinality of a fuzzy set, also called as its sigma- count is given by

Definition 2.6: The non-fuzzy cardinality of a fuzzy set $\mathrm{A}$ given by $A=\left\{\left(x_{1}, \mu_{1}\right),\left(x_{2}, \mu_{2}\right), \ldots\left(x_{n}, \mu_{n}\right)\right\}$ is given by $\Sigma \operatorname{Count}(A)=\sum_{i=1}^{n} \mu_{i}$

The less simple but a more natural extension, which is a fuzzy number introduced by Zadeh [ ] is defined as

Definition 2.7: For a fuzzy set A defined over a universe $\mathrm{U}=\left\{x_{1}, x_{2}, \ldots x_{n}\right\}$ is defined as

$$
A=\sum_{\alpha} \alpha A_{\alpha}
$$

where $A_{\alpha}=\left\{x_{i} \mid \mu_{A}\left(x_{i}\right) \geq \alpha\right\}, 0 \leq \alpha \leq 1$.
Here, $\Sigma$ stands for the union and $\alpha A_{\alpha}$ is a fuzzy set whose membership function is defined by

$$
\mu_{\alpha A_{\alpha}}(x)=\left\{\begin{array}{l}
\alpha, \text { for } \mathrm{x} \in A_{\alpha} \\
0, \text { elsewhere }
\end{array}\right.
$$

Definition 2.8: Let $\mathrm{A}$ be as defined in definition 2.5. Then the fuzzy count can be defined as

FGcount $(A):[0,1] \rightarrow N$, where $N$ is the set of natural numbers and

$$
\begin{aligned}
F G \operatorname{count}(A)(\alpha) & =\left|\mathrm{A}_{\alpha}\right|, \alpha \in[0,1] \\
& =\left|\left\{x: \mu_{A}(x) \geq \alpha\right\}\right|
\end{aligned}
$$

By definition FG count(A) is a bag on $[0,1]$.

Definition 2.9: We define the fuzy lower count of $A$ as $\operatorname{FLcount}(\mathrm{A})(\alpha):[0,1] \rightarrow \mathrm{N}$ such that

$$
\begin{aligned}
\operatorname{FLcount}(\mathrm{A})(\alpha) & =\left|\left\{\mathrm{x}: \mu_{A}(x) \leq \alpha\right\}\right| \\
& =\left|\left\{x: \mu_{A^{C}}(x) \geq \alpha\right\}\right| \\
& =\mathrm{FG} \operatorname{Gcount}\left(\mathrm{A}^{\mathrm{C}}\right)(1-\alpha) ;
\end{aligned}
$$

Where $A^{C}$ denotes the complement of the fuzzy set A.

Definition 2.10: The fuzzy equality count of a fuzzy set $\mathrm{A}$ is denoted by FE count (A) and is defined as

$$
\begin{aligned}
\operatorname{FEcount}(\mathrm{A})(\alpha)= & \left|\left\{\mathrm{x}: \mu_{\mathrm{A}}(\mathrm{x})=\alpha\right\}\right| \\
& =\mathrm{FG} \operatorname{count}(\mathrm{A})(\alpha) \cap \mathrm{FLcount}(\mathrm{A})(\alpha)
\end{aligned}
$$

for each $\alpha \in[0,1]$.

Out of the above three definitions of count of fuzzy sets, the extension of definition 2.5 is straight forward and that of definition 2.6 is due to Tripathy et al [9]. In this paper we consider the third case and extend it to the context of intuitionistic fuzzy sets and prove many of its properties.

\section{CARDINALITY OF INTUITIONISTIC FUZZY SETS}

In this section, we introduce the concept of cardinality of intuitionistic fuzzy sets using bag theoretic concepts.

Let us assume an intuitionistic fuzzy set A defined over a universe of discourse $\mathrm{X}$ us represented as-

$$
\mathrm{A}=\sum_{i=1}^{n}\left(x_{i}, \mu_{i}(x), v_{i}(x)\right) \text { where } X=\left\{x_{1}, x_{2}, \ldots, x_{n}\right\}
$$

Let us define

$$
\mathrm{J}=\{(\alpha, \beta) \in[0,1] \times[0,1] \mid 0 \leq \alpha+\beta \leq 1\} .
$$

Definition 3.1: Let $\mathrm{A}$ be an intuitionistic fuzzy set on a universe $\mathrm{U}$. Then for any $(\alpha, \beta) \in J$, we define IFGCount(A) as 


$$
\begin{aligned}
\operatorname{IFGCount}(\mathrm{A}) & (\alpha, \beta)=\left|A_{\alpha, \beta}\right| \\
= & \mid\left\{\mathrm{x}: \mu_{A}(x) \geq \alpha \text { and } v_{A}(x) \leq \beta\right\} \mid
\end{aligned}
$$

Here IFGCount(A) is a bag on $J$.

Note3.1: When $A$ is a fuzzy set, we have $v_{A}(x)=1-\mu_{A}(x)$. Also, we note that $A_{\alpha, 1-\alpha}=A_{\alpha}$.

For, $\quad A_{\alpha, 1-\alpha}=\left\{x: \mu_{A}(x) \geq \alpha, v_{A}(x) \leq 1-\alpha\right\}=$ $\left\{x: \mu_{A}(x) \geq \alpha, 1-\mu_{A}(x) \leq 1-\alpha\right\}=\left\{x: \mu_{A}(x) \geq \alpha\right\}=A_{\alpha}$

Hence, IFGCount(A) $(\alpha, 1-\alpha)=\operatorname{FGCount}(\mathrm{A})(\alpha)$. This implies that FGCount(A) is a special case of IFGCount(A).

Definition 3.2: Let $\mathrm{A}$ be an intuitionistic fuzzy set on a universe $\mathrm{U}$. Then for any $(\alpha, \beta) \in J$, we define IFLCount(A) as

$\operatorname{IFLCount}(\mathrm{A})(\alpha, \beta)=\mid\left\{\mathrm{x}: \mu_{A}(x) \leq \alpha\right.$

and $\left.v_{A}(x) \geq \beta\right\}|=|\left\{\mathrm{x}: v_{A}(x) \geq \beta\right.$

and $\left.\mu_{A}(x) \leq \alpha\right\}|=| A_{\beta, \alpha}^{C} \mid=\operatorname{IFGCount}\left(A^{C}\right)(\beta, \alpha)$

Definition 3.3: Let $\mathrm{A}$ be an intuitionistic fuzzy set on a universe $\mathrm{U}$. Then for any $(\alpha, \beta) \in J$, we define IFECount(A) as

$\operatorname{IFECount}(\mathrm{A})(\alpha, \beta)=\mid\left\{\mathrm{x}: \mu_{A}(x)=\alpha\right.$ and $\left.v_{A}(x)=\beta\right\} \mid$

\section{EXAMPLE 3.1:}

Let $\mathrm{U}=\{\mathrm{a}, \mathrm{b}, \mathrm{c}, \mathrm{d}, \mathrm{e}, \mathrm{f}\}$ and

$A=\{(a, 0.5,0.4)+(b, 0.8,0.1)+(c, 0.2,0.6)+(d, 0.5,0.1)\}$.

Then

$$
\begin{aligned}
& A_{(0.8,0.1)}=\{b\}, A_{(0.8,0.4)}=\{b\}, A_{(0.8,0.6)}=\{b\}, \\
& A_{(0.5,0.1)}=\{b, d\}, A_{(0.5,0.4)}=\{a, b, d\}, \\
& A_{(0.5,0.6)}=\{a, b, d\}, A_{(0.2,0.1)}=\{b, d\}, \\
& A_{(0.2,0.4)}=\{a, b, d\} \text { and } A_{(0.2,0.6)}=\{a, b, c, d\} .
\end{aligned}
$$

So,

$\operatorname{IFGCount}(\mathrm{A})=(0.8,0.1) / 1+(0.8,0.4) / 1+(0.8,0.6) / 1+$ $(0.5,0.1) / 2+(0.5,0.4) / 3+(0.5,0.6) / 3+(0.2,0.1) / 2+$ $(0.2,0.4) / 3+(0.2,0.6) / 4$.

Now,

$$
\begin{aligned}
\mathrm{A}^{C}=\{ & (a, 0.4,0.5)+(b, 0.1,0.8) \\
& +(c, 0.6,02)+(d, 0.1,0.5)\}
\end{aligned}
$$

So,
$\operatorname{IFLCount}(\mathrm{A})=(0.8,0.1) / 4+(0.8,0.4) / 2+(0.8,0.6) / 1+$ $(0.5,0.1) / 3+(0.5,0.4) / 2+(0.5,0.6) / 1+(0.2,0.1) / 1+$ $(0.2,0.4) / 1+(0.2,0.6) / 1$.

$\operatorname{IFECount}(\mathrm{A})=(0.8,0.1) / 1+(0.8,0.4) / 0+(0.8,0.6) / 0+$ $(0.5,0.1) / 1+(0.5,0.4) / 1+(0.5,0.6) / 0+(0.2,0.1) / 0+$ $(0.2,0.4) / 0+(0.2,0.6) / 1$.

NOTE 3.2: Let A be an intuitionistic fuzzy set on a universe $\mathrm{U}$. Then for any $(\alpha, \beta) \in J$, IFECount(A) $(\alpha, \beta)=\operatorname{IFGCount}(\mathrm{A})(\alpha, \beta) \quad \cap \operatorname{IFLCount}(\mathrm{A})$ $(\alpha, \beta)$.

Proof: By the definitions of the three concepts of intuitionistic fuzzy greater count, intuitionistic fuzzy lower count and intuitionistic fuzzy equal count we have LHS is a subset of each of the components in the RHS and hence is a subset of their intersection. Also,

$$
\begin{aligned}
& x \in \operatorname{IFGCount}(A)(\alpha, \beta) \cap \operatorname{IFLCount}(A)(\alpha, \beta) \\
& \Rightarrow \mu_{A}(x) \geq \alpha \text { and } v_{A}(x) \leq \beta \text { and } \mu_{A}(x) \leq \alpha \\
& \text { and } v_{A}(x) \geq \beta \\
& \Rightarrow \mu_{A}(x)=\alpha \text { and } v_{A}(x)=\beta \\
& \Rightarrow x \in \operatorname{IFECount}(\mathrm{A})(\alpha, \beta)
\end{aligned}
$$

So RHS is a subset of the LHS. This completes the proof.

Before establishing the next theorem, we define an ordering of elements on $\mathrm{J}$, which is a partially ordered relation.

Definition 3.4: For any two elements $(\alpha, \beta)$ and $(\gamma, \delta) \in J$, we define $(\alpha, \beta) \succ(\gamma, \delta)$ if and only if $\alpha \geq \gamma$ and $\beta \leq \delta$.

Note 3.3: Then $\succ$ is a partially ordered relation over $J$.

(i) Clearly, $(\alpha, \beta) \succ(\alpha, \beta)$ by definition.

(ii) Also, $(\alpha, \beta) \succ(\gamma, \delta)$ and $(\gamma, \delta) \succ(\alpha, \beta)$

$\Rightarrow \alpha \geq \gamma, \beta \leq \delta$ and $\gamma \geq \alpha, \delta \leq \beta$.

So that $\alpha=\gamma$ and $\beta=\delta$. Hence, $(\alpha, \beta)=(\gamma, \delta)$.

From this it follows that $\succ$ is antisymmetric.

Finally,

(iii) $(\alpha, \beta) \succ(\gamma, \delta)$ and $(\gamma, \delta) \succ(u, v) \Rightarrow \alpha \geq \gamma$, $\beta \leq \delta$ and $\gamma \geq u, \delta \leq v \Rightarrow \alpha \geq u, \beta \leq v$.

So that $(\alpha, \beta) \succ(u, v)$ and hence $\succ$ is transitive.

Theorem 3.1: IFGCount(A) and IFLCount(A) are decreasing functions on $J$ for any IFS A.

Proof:

Now, $(\alpha, \beta) \succ(\gamma, \delta) \Rightarrow \alpha \geq \gamma, \beta \leq \delta$. So, 
$\operatorname{IFGCount(A)} \quad(\alpha, \beta) \quad=\mid\left\{\mathrm{x}: \quad \mu_{A}(x) \geq \alpha \quad\right.$ and $\left.v_{A}(x) \leq \beta \quad\right\}|\leq|\left\{x: \mu_{A}(x) \geq \gamma, v_{A}(x) \leq \delta\right\} \mid=$ IFGCount (A) $(\alpha, \beta)$

The proof for IFLCount(A) is similar.

\section{Properties of InTUITIONISTIC FuZZY COUNTS}

In this section, we establish some properties of the three fuzzy counts.

Theorem 4.1: For any two intuitionistic fuzzy sets A and $\mathrm{B}$ on $\mathrm{X}$

(i)If $\mathrm{A} \subseteq \mathrm{B}$ then $\operatorname{IFGCount}(A) \subseteq \operatorname{IFGCount}(B)$

(ii) $\operatorname{IFGCount}(A) \cup \operatorname{IFGCount}(B) \subseteq \operatorname{IFGCount}(A \cup B)$

(iii) $\operatorname{IFGCount}(A \cap B) \subseteq \operatorname{IFGCount}(A) \cap \operatorname{IFGCount}(B)$

Proof:

(i) Suppose $A \subseteq B$. Then, for any $\alpha, \beta \quad[0,1]$

$\operatorname{IFGCount}(\mathrm{A})(\alpha, \beta)$

$=\mid\left\{x: \mu_{\mathrm{A}}(x) \geq \alpha\right.$ and $\left.v_{\mathrm{A}}(x) \leq \beta\right\} \mid$

$\leq \mid\left\{x: \mu_{\mathrm{B}}(x) \geq \alpha\right.$ and $\left.\left.v_{\mathrm{B}}(x) \leq \beta\right\}\right\}$

Hence, IFGCount(A) $\subseteq$ IFGCount(B)

(ii) For any $\alpha, \beta \in[0,1] \quad$ (IFGCount(A) $\cup$ $\operatorname{IFGCount}(\mathrm{B}))(\alpha, \beta)$

$=\max \{\operatorname{IFGCount}(\mathrm{A})(\alpha, \beta), \operatorname{IFGCount}(\mathrm{B})(\alpha, \beta)\}$

$\subseteq \max \{\operatorname{IFGCount}(\mathrm{A} \cup \mathrm{B})(\alpha, \beta)$,

$\operatorname{IFGCount}(\mathrm{A} \cup \mathrm{B})(\alpha, \beta)\}=\operatorname{IFGCount}(\mathrm{A} \cup \mathrm{B})(\alpha, \beta)$

(iii)(IFGCount(A) $\bigcap \operatorname{IFGCount(B))(\alpha ,\beta )}$

$=\min \{\operatorname{IFGCount}(\mathrm{A})(\alpha, \beta), \operatorname{IFGCount}(\mathrm{B})(\alpha, \beta)\}$

$\supseteq \min \{\operatorname{IFGCount}(\mathrm{A} \bigcap \mathrm{B})(\alpha, \beta), \operatorname{IFGCount}(\mathrm{A} \bigcap \mathrm{B})$

$(\alpha, \beta)\}=\operatorname{IFGCount}(\mathrm{A} \bigcap \mathrm{B})(\alpha, \beta)$.

This completes the proof.

Theorem 4.2: For any two Intuitionistic fuzzy subsets $\mathrm{A}, \mathrm{B}$ on a finite set $\mathrm{X}$, we have

$(i)(\operatorname{IFGCOunt}(A) \cup \operatorname{IFGCount}(B)) \oplus$

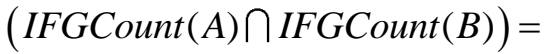

$\operatorname{IFGCount}(A) \oplus \operatorname{IFGCount}(B)$

(ii)IFGCount $(A \cup B) \oplus \operatorname{IFGCount}(A \cap B)$

$\supseteq \operatorname{IFGCount}(A) \oplus \operatorname{IFGCount}(B)$

Proof:

Proof of (i)

For any $(\alpha, \beta) \in \mathbf{J}$, by (2.13) above
$\left(\begin{array}{l}(\operatorname{IFGCOunt}(A) \cup \operatorname{IFGCOunt}(B)) \oplus \\ (\operatorname{IFGCOunt}(A) \cap \operatorname{IFGCOunt}(B))\end{array}\right)(\alpha, \beta)$

$=(\operatorname{IFGCOunt}(A) \cup \operatorname{IFGCount}(B))(\alpha, \beta)+$

$(\operatorname{IFGCount}(A) \cap \operatorname{IFGCount}(B))(\alpha, \beta)$

Now by (2.11) and (2.12) above,

$\max \{(\operatorname{IFGCount}(A))(\alpha, \beta),(\operatorname{IFGCOunt}(B))(\alpha, \beta)\}+$ $\min \{(\operatorname{IFGCount}(A))(\alpha, \beta),(\operatorname{IFGCount}(B))(\alpha, \beta)\}=$ $(\operatorname{IFGCount}(A))(\alpha, \beta)+(\operatorname{IFGCOunt}(B))(\alpha, \beta)$

since one of the two is the maximum and the other is the minimum.

This completes the proof.

Proof of (ii): Let $\mathrm{X}=\left\{x_{1}, x_{2}, \ldots, x_{n}\right\}$ be the universe of discourse and $\mathrm{A}$ and $\mathrm{B}$ be two Intuitionistic fuzzy subsets on $\mathrm{X}$. Then for any $(\alpha, \beta) \in \mathrm{J}$, the following 16 cases arise. We enumerate these cases along with their cardinalities. The number of elements of each of the categories is provided in the braces following the case heading:

Case-1:( $\left.\mathrm{r}_{1}\right)$

$\left(\mu_{A}(x) \geq \alpha, v_{A}(x) \leq \beta\right) \&\left(\mu_{B}(x) \geq \alpha, v_{B}(x) \leq \beta\right)$

Case 2:( $\left.\mathrm{r}_{2}\right)$

$\left(\mu_{A}(x) \geq \alpha, v_{A}(x) \leq \beta\right) \&\left(\mu_{B}(x) \geq \alpha, v_{B}(x)>\beta\right)$

Case 3: $\left(\mathrm{r}_{3}\right)$

$\left(\mu_{A}(x) \geq \alpha, v_{A}(x)>\beta\right) \&\left(\mu_{B}(x) \geq \alpha, v_{B}(x) \leq \beta\right)$

Case 4: $\left(\mathrm{r}_{4}\right)$

$\left(\mu_{A}(x) \geq \alpha, v_{A}(x)>\beta\right) \&\left(\mu_{B}(x) \geq \alpha, v_{B}(x)>\beta\right)$

Case 5: $\left(\mathrm{s}_{1}\right)$

$\left(\mu_{A}(x) \geq \alpha, v_{A}(x) \leq \beta\right) \&\left(\mu_{B}(x)<\alpha, v_{B}(x) \leq \beta\right)$

Case 6: $\left(\mathrm{s}_{2}\right)$

$\left(\mu_{A}(x) \geq \alpha, v_{A}(x) \leq \beta\right) \&\left(\mu_{B}(x)<\alpha, v_{B}(x)>\beta\right)$

Case $7:\left(\mathrm{s}_{3}\right)$

$\left(\mu_{A}(x) \geq \alpha, v_{A}(x)>\beta\right) \&\left(\mu_{B}(x)<\alpha, v_{B}(x) \leq \beta\right)$

Case 8: $\left(\mathrm{s}_{4}\right)$

$\left(\mu_{A}(x) \geq \alpha, v_{A}(x)>\beta\right) \&\left(\mu_{B}(x)<\alpha, v_{B}(x)>\beta\right)$

Case 9: $\left(\mathrm{t}_{1}\right)$

$\left(\mu_{A}(x)<\alpha, v_{A}(x) \leq \beta\right) \&\left(\mu_{B}(x) \geq \alpha, v_{B}(x) \leq \beta\right)$

Case 10: $\left(\mathrm{t}_{2}\right)$

$\left(\mu_{A}(x)<\alpha, v_{A}(x) \leq \beta\right) \&\left(\mu_{B}(x) \geq \alpha, v_{B}(x)>\beta\right)$ 
Case 11: $\left(\mathrm{t}_{3}\right)$

$\left(\mu_{A}(x)<\alpha, v_{A}(x)>\beta\right) \&\left(\mu_{B}(x) \geq \alpha, v_{B}(x) \leq \beta\right)$

Case 12: $\left(\mathrm{t}_{4}\right)$

$\left(\mu_{A}(x)<\alpha, v_{A}(x)>\beta\right) \&\left(\mu_{B}(x) \geq \alpha, v_{B}(x)>\beta\right)$

Case 13: $\left(\mathrm{u}_{1}\right)$

$\left(\mu_{A}(x)<\alpha, v_{A}(x) \leq \beta\right) \&\left(\mu_{B}(x)<\alpha, v_{B}(x) \leq \beta\right)$

Case 14: $\left(\mathrm{u}_{2}\right)$

$\left(\mu_{A}(x)<\alpha, v_{A}(x) \leq \beta\right) \&\left(\mu_{B}(x)<\alpha, v_{B}(x)>\beta\right)$

Case 15: $\left(\mathrm{u}_{3}\right)$

$\left(\mu_{A}(x)<\alpha, v_{A}(x)>\beta\right) \&\left(\mu_{B}(x)<\alpha, v_{B}(x) \leq \beta\right)$

Case 16: $\left(\mathrm{u}_{4}\right)$

$\left(\mu_{A}(x)<\alpha, v_{A}(x)>\beta\right) \&\left(\mu_{B}(x)<\alpha, v_{B}(x)>\beta\right)$

Now,

IFGCount $(A \cup B)(\alpha, \beta)$

$=\left|\left\{x: \mu_{A \cup B}(x) \geq \alpha, v_{A \cup B}(x) \leq \beta\right\}\right|$

$=\left|\begin{array}{c}\left\{x: \max \left\{\mu_{A}(x), \mu_{B}(x)\right\} \geq \alpha\right. \\ \left.\& \min \left\{v_{A}(x), v_{B}(x)\right\} \leq \beta\right\}\end{array}\right|$

$=\left|\begin{array}{c}\left\{x:\left\{\mu_{A}(x) \geq \alpha \text { or } \mu_{B}(x) \geq \alpha\right\}\right. \\ \left.\&\left\{v_{A}(x) \leq \beta \text { or } v_{B}(x) \leq \beta\right\}\right\}\end{array}\right|$

$=\mid$ Case $-1|+|$ Case $-2|+|$ Case $-5|+|$ Case $-6|+|$ Case $-9|+|$ Case$10|+|$ case $-3|+|$ Case- $7|+|$ Case-11|

$=r_{1}+r_{2}+r_{3}+s_{1}+s_{2}+s_{3}+t_{1}+t_{2}+t_{3}$

$\operatorname{IFGCount}(A \cap B)(\alpha, \beta)=\left|\begin{array}{r}\left\{x: \mu_{A \cap B}(x) \geq \alpha,\right. \\ \left.v_{A \cap B}(x) \leq \beta\right\}\end{array}\right|$

$=\left|\begin{array}{c}\left\{x: \min \left\{\mu_{A}(x), \mu_{B}(x)\right\} \geq \alpha\right. \\ \left.\& \max \left\{v_{A}(x), v_{B}(x)\right\} \leq \beta\right\}\end{array}\right|$

$=\left|\begin{array}{c}\left\{x:\left\{\mu_{A}(x) \geq \alpha \text { and } \mu_{B}(x) \geq \alpha\right\}\right. \\ \left.\&\left\{v_{A}(x) \leq \beta \text { and } v_{B}(x) \leq \beta\right\}\right\}\end{array}\right|$

$=\mid$ Case $-1 \mid$

$=r_{1}$

So,

IFGCount $(A \cup B)(\alpha, \beta)+\operatorname{IFGCount}(A \cap B)(\alpha, \beta)$

$=2 r_{1}+r_{2}+r_{3}+s_{1}+s_{2}+s_{3}+t_{1}+t_{2}+t_{3}$

Again,

IFGCount $\quad(A)(\alpha, \beta) \quad=\left\{\mathrm{x}: \quad \mu_{A}(x) \geq \alpha \quad\right.$ and $\left.v_{A}(x) \leq \beta\right\} \mid$

$=\mid$ Case- $1|+|$ Case $-2|+|$ Case $-5|+|$ case $-6 \mid$ $=r_{1}+r_{2}+s_{1}+s_{2}$

IFGCount $(B)(\alpha, \beta)=\mid\left\{\mathrm{x}: \quad \mu_{B}(x) \geq \alpha \quad\right.$ and $\left.v_{B}(x) \leq \beta\right\} \mid$

$=\mid$ Case- $1|+|$ Case $-3|+|$ Case- $-9|+|$ Case $-11 \mid$

$=r_{1}+r_{3}+t_{1}+t_{3}$

So, $\operatorname{IFGCount}(A)(\alpha, \beta)+\operatorname{IFGCount}(B)(\alpha, \beta)$

$=2 r_{1}+r_{2}+r_{3}+s_{1}+s_{2}+t_{1}+t_{3}$

From $(*)$ and $(* *)$ the result follows.

Corollary 4.1: It is clear from $(*)$ and $(* *)$ that the cases when $s_{3} \neq 0$ or $t_{2} \neq 0$ the equality does not hold. So, necessary and sufficient conditions for the equality to hold are $s_{3}=0$ and $t_{2}=0$.

NOTE 4.1: It may be noted that in the fuzzy case the equality holds. Here, we find that

$$
\begin{gathered}
s_{3}=\mid \begin{array}{r}
\left(\mu_{A}(x) \geq \alpha, v_{A}(x)>\beta\right) \\
\&\left(\mu_{B}(x)<\alpha, v_{B}(x) \leq \beta\right)
\end{array} \\
\beta=1-\alpha . \text { So, } \mu_{A}(x) \geq \alpha \\
\Rightarrow v_{A}(x)=1-\mu_{A}(x) \leq 1-\alpha=\beta .
\end{gathered}
$$

Hence, $s_{3}=|\phi|=0$. Also,

$$
t_{2}=\left|\begin{array}{r}
\left(\mu_{A}(x)<\alpha, v_{A}(x) \leq \beta\right) \\
\&\left(\mu_{B}(x) \geq \alpha, v_{B}(x)>\beta\right)
\end{array}\right| \text { In this case, }
$$$$
\beta=1-\alpha \text {. So, } \mu_{A}(x)<\alpha
$$$$
\Rightarrow v_{A}(x)=1-\mu_{A}(x)>1-\alpha=\beta \text {. }
$$

Hence, $t_{2}=|\phi|=0$.

So, the conditions for equality are satisfied.

NOTE 4.2: Since union, intersection and $\oplus$ of bags are commutative operations, we get

(i) IFGCount $(A) \cup I F G C \operatorname{Count}(B)=$ IFGCount $(B) \cup I F G \operatorname{Count}(A)$

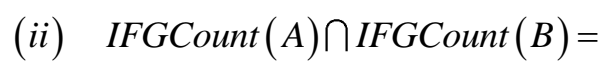
IFGCount $(B) \cap I F G C$ Count $(A)$

(iii) $\operatorname{IFGCount}(A) \oplus \operatorname{IFGCount}(B)=$ $\operatorname{IFGCount}(B) \oplus \operatorname{IFGCount}(A)$

NOTE 4.3: From ([4] Theorem 1.1(v)) it follows that

(i) IFGCount $(A \cap B) \subseteq I F G C \operatorname{Count}(A)$

$$
\subseteq I F G \operatorname{Count}(A \cup B)
$$

Directly from ([4] Theorem 1.1(vi)) we get, 


$$
\begin{aligned}
& \text { (ii) } I F G \operatorname{Count}(A) \Theta I F G \operatorname{Count}(B) \subseteq \\
& I F G \operatorname{Count}(A) \subseteq I F G \operatorname{Count}(A) \oplus I F G \operatorname{Count}(B)
\end{aligned}
$$

NOTE 4.4: From ([4] Theorem 1.3) it follows that for any three fuzzy subsets $\mathrm{A}, \mathrm{B}, \mathrm{C}$ on $\mathrm{X}$

$$
\begin{aligned}
& \text { (i) } \operatorname{IFGCount}(A) \cup(\operatorname{IFGCount}(B) \cap \operatorname{IFGCount}(\mathrm{C}))= \\
& (\operatorname{IFGCount}(\mathrm{A}) \cup \operatorname{IFGCount}(\mathrm{B})) \cap \\
& (\operatorname{IFGCount}(\mathrm{A}) \cup \operatorname{IFGCount}(\mathrm{C}))
\end{aligned}
$$

(ii) $\operatorname{IFGCount}(\mathrm{A}) \cap(\operatorname{IFGCount}(\mathrm{B}) \cup \operatorname{IFGCount}(\mathrm{C}))=$ (IFGCount $(\mathrm{A}) \cap \operatorname{IFGCount}(\mathrm{B})) \cup$ (IFGCount $(\mathrm{A}) \cap \operatorname{IFGCount}(\mathrm{C}))$

NOTE 4.5: From ([4] Theorem 1.4) it follows that for any three fuzzy subsets $\mathrm{A}, \mathrm{B}$ and $\mathrm{C}$ on $\mathrm{X}$

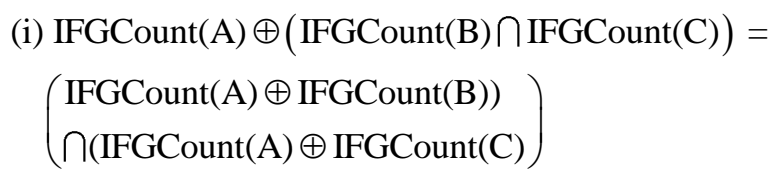

(ii) $\operatorname{IFGCount}(\mathrm{A}) \oplus(\operatorname{IFGCount}(\mathrm{B}) \bigcup \operatorname{IFGCount}(\mathrm{C}))=$ $\left(\begin{array}{l}\operatorname{IFGCount}(\mathrm{A}) \oplus \operatorname{IFGCount}(\mathrm{B})) \bigcup \\ (\mathrm{IFGCount}(\mathrm{A}) \oplus \operatorname{IFGCount}(\mathrm{C})\end{array}\right)$

NOTE 4.6: From ([4] Theorem 1.5) it follows that for any three fuzzy subsets A, B, C on X

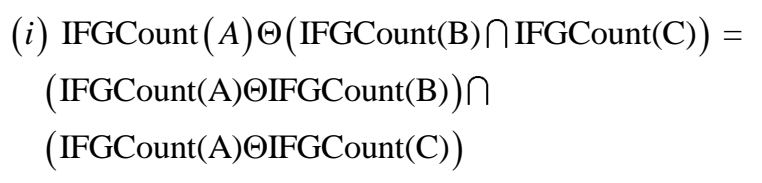
(ii) $\operatorname{IFGCount}(\mathrm{A}) \Theta(\operatorname{IFGCount}(\mathrm{B}) \bigcup \operatorname{IFGCount}(\mathrm{C}))=$

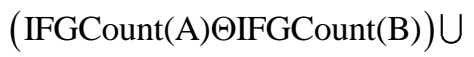

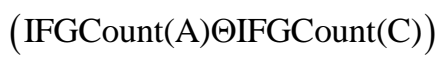

\section{CONCLUSION}

Finding the cardinality of a fuzzy set is an interesting topic and there were three different approaches to define this due to Deluca and Termini, Zadeh and Tripathy et al. Since intuitionistic fuzzy sets are generalizations of fuzzy sets recently their cardinality was obtained as an extension of the definition for fuzzy sets given by Deluca and termini. In this paper, we provided one more definition of cardinality of an intuitionistic fuzzy set as an extension of the bag theoretic approach of Tripathy et al. Also, we establish many of their properties like- the cardinality of sets, which are used in order estimation of complexities of algorithms, the cardinality of fuzzy sets and that for intuitionistic fuzzy sets can be applied for the order of estimation of complexities of fuzzy and intuitionistic algorithms and interpretation of test score semantics.

\section{REFERENCES}

[1] Zadeh, L. A.: Fuzzy Sets, Information and Control, vol.8, (1965), pp.338-353

[2] Klir, G. J. and Yuan, B.: Fuzzy Sets and Fuzzy Logic, Theory and Applications, Prentice Hall of India pvt. Ltd., New Delhi, (1997).

[3] DeLuca, A. and Termini, S.: A definition of a nonprobabilistic entropy in the setting of Fuzzy Set Theory, Information and Control, Vol.20, (1972), pp.301-302.

[4] Zadeh, L. A.: Probability measures of fuzzy events, Journal of Mathematical Analysis and Applications, vol.23, (1968), pp.421-427.

[5] Zadeh, L.A.: test-score semantics for Natural Languages and meaning representation via PRUF, in: Rieger, B. (ed.), Empirical Semantics, Brockmeyer, Bochum, Germany, (1982), pp. 281-349.

[6] Mizumoto, M. and Tanaka, K.: Some Properties of fuzzy numbers, in: Advances in Fuzzy Set Theory and Applications, eds.M.M.Gupta, R.K.Ragade and R.R.Yager, North Holland, Amsterdam, (1979), pp.153-164.

[7] Atanassov, K.T.: intuitionistic fuzzy sets, Fuzzy Sets and Systems, vol.20, (1986), pp.87-96.

[8] Tripathy, B.K., Jena, S.P. and Ghosh, S.K.: An Intuitionistic fuzzy count and cardinality of Intuitionistic fuzzy sets, Malaya Journal of Matematik, 4(1), (2013), pp. 123-133.

[9] Cerf, V., Fernandez, E., Gostalow, K. and Volausky, S.: Formal control and low properties of a model of computation, report ENG, 7178, Computer Science Dept. of California, Los-Angeles, California, Dec (1971), pages 81.

[10] Jena, S.P., Ghosh, S.K. and Tripathy, B. K.: On the theory of bags and lists, Information Science, vol.132, (2001), pp.241-254.

[11] Peterson, J.: Computation sequence spaces, Journal of Computer and System Sciences, vol.13, no.1, Aug. (1976), pp.1-24.

[12] Yager, R.R.: On the theory of bags, International Journal of General Systems, 13, (1986), pp.23-37

[13] Tripathy, B.K., Jena, S.P. and Ghosh, S.K.: A Bag Theoretic Fuzzy Count and Cardinality of Fuzzy Sets, 2013 IEEE ICCIC, Madurai, Dec.26-28, (2013), pp.379 384.

[14] Chakraborty, K., Biswas, R, and Nanda, S.: On Yager's theory of bags and fuzzy bags, Computer and Artificial Intelligencs, 18, (1999), pp. 1-17.

\section{Authors' Profiles}

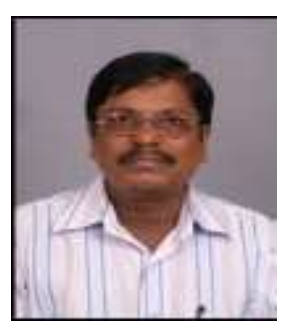

B.K.Tripathy is working as a senior professor in SCSE of VIT University, India. So far, he has produced $16 \mathrm{PhDs}$, 13 M.Phils and 2 M.S (By research) candidates. He has published around 220 technical articles in different international journals/proceedings of international conferences/ Edited research volumes. Also, he has edited two research volumes. He is a life member/member/senior member of 20 international professional bodies like the ACM, IEEE, CSI, ACEEE, IAENG, IMS, OMS and IRSS. He is in the editorial Board/ reviewer of more than 50 international journals. His current research interest includes Rough Sets and Knowledge Engineering, Fuzzy Sets and systems, Data Mining, Soft Computing, Social Networks, E-Learning, Image 
Processing, List Theory, Theory of Multisets, Soft Set theory and Applications and Remote Laboratories.

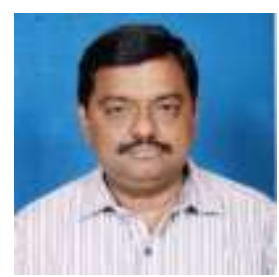

M.K. Satapathy is working as a lecturer in the R.G. College, Padmapur, Odisha. $\mathrm{He}$ is pursuing his Ph.D. degree under the supervision of Dr.B.K. Tripathy. His research interest includes rough set theory, fuzzy set theory and applications. He has 5 publications to his credit.

S.Khandelwal is a graduate in computing science and engineering from VIT University, India. Presently he is working with Flipkart. His research interest includes soft computing and fuzzy set theory.

How to cite this paper: B.K.Tripathy, S.Khandelwal, M.K.Satapathy,"A Bag Theoretic Approach towards the Count of an Intuitionistic Fuzzy Set", International Journal of Intelligent Systems and Applications (IJISA), vol.7, no.5, pp.16-23, 2015. DOI: 10.5815/ijisa.2015.05.03 